\title{
El mapuzungun en niños mapuche de La Araucanía. Reflexiones sobre adquisición de la lengua a partir de un estudio de medición directa*
}

\author{
Fernando Wittig González** \\ Paula Alonqueo Boudon***
}

\section{Resumen}

Se presentan los resultados de una investigación que describe la competencia en mapuzungun de niños de La Araucanía. Se aplicó un cuestionario de elaboración ad hoc que incluyó ejercicios lingüísticos, lenguaje corporal y tareas cognitivas con pertinencia cultural. Participaron 99 niños, entre 6 y 10 años, procedentes de 4 escuelas rurales. Los resultados muestran desempeños generales de nivel bajo, con mejores resultados en comprensión que en producción. Se identificaron casos singulares de niños entre 8 y 10 años con competencia media y alta. A partir de estos hallazgos, se plantean reflexiones preliminares sobre la adquisición del mapuzungun en población infantil rural.

Palabras Clave: Mapuzungun, competencia lingüística, niños mapuche, cuestionario, adquisición.

\section{Mapuzungun Competence in Mapuche Children. Issues about Language Acquisition in la araucania, Chile}

\begin{abstract}
This article describes the mapuzungun competence of children from La Araucanía region, Chile. The study included 99 children, aged 6 to 10 years, from 4 rural schools. The questionnaire considered linguistic exercises, body language and cognitive tasks with cultural relevance. Results show a low level competence, with better results in comprehension than in production. Single cases of children between 8 and 10 years old with medium and high competence levels were identified. Based on these findings, some issues on mapuzungun acquisition in the rural child population are presented.
\end{abstract}

Keywords: Mapuzungun, Linguistic competence, Mapuche children, Questionnaire, Acquisition.

\footnotetext{
Este trabajo se enmarca dentro del Proyecto Fondecyt No ${ }^{\circ} 1130504$ Factores culturales, evolutivos y socio-lingüísticos en la construcción de los conceptos de espacio, tiempo, y número en escolares mapuche de zonas rurales.

** Chileno. Doctor en Lingüística de la Universidad de Concepción, Chile. Académico adscrito al Departamento de Lenguas y al Núcleo de Investigación en Estudios Interculturales e Interétnicos (NEII) de la Universidad Católica de Temuco, Temuco, Chile, fwittig@uct.cl

*** Chilena. Doctora en Psicología Evolutiva de la Universidad Autónoma de Madrid, España. Académica adscrita al Departamento de Psicología y al Centro de Investigación en Lenguas, Cognición y Cultura (CILCC) de la Universidad de La Frontera, Temuco, Chile. paula.alonqueo@ufrontera.cl
} 


\section{Introducción}

Desde hace un tiempo parece haber plena coincidencia entre los investigadores en la definición del estado del mapuzungun como lengua en peligro. Las preocupaciones sobre la vitalidad de la lengua han transitado desde los diagnósticos generales hacia propuestas de revitalización que incluyen la con participación de distintos actores del mundo mapuche, la academia, organismos técnicos nacionales e internacionales. Los esfuerzos por revertir esta situación se fundamentan en la disminución generalizada de hablantes, con especial énfasis en la escaza generación de nuevos hablantes entre la población infantil. En otras palabras, se puede afirmar que la continuidad de la lengua mapuche, no solo como instrumento de comunicación, sino también como depositario y reflejo de la cultura e identidad del Pueblo Mapuche, depende en gran medida del desarrollo de competencias lingüísticas y comunicativas en los niños mapuches (Zúñiga \& Olate, 2017).

En contraste con lo anterior, el conocimiento sobre los procesos de adquisición y aprendizaje del mapuzungun en población infantil es aún preliminar. Por extensión, cuestiones más específicas como la descripción de niveles de competencia y modalidades de uso en la población infantil solo se han abordado de manera tangencial. Dentro de los estudios disponibles, Gundermann, Canihuan, Clavería y Faúndez (2009; 2011), Lagos (2012), Henríquez (2014, 2016); Henríquez y Salamanca (2015); Zúñiga (2007) y Zúñiga y Olate (2017) constituyen valiosos aportes para descripciones generales y específicas de la situación sociolingüística del mapuzungun. No obstante, los datos relativos a tipos de hablante de diferentes edades, niveles de competencia y ámbitos de uso se obtienen sobre la base de la percepción o autorreporte de los sujetos de estudio. Por otro lado, los trabajos de Quilaqueo y Quintriqueo (2010); Catriquir y Durán (2007); Quidel y Pichinao (2007) describen procesos socioeducativos tradicionales que constituyen lo que los autores denominan 'modelo educativo mapuche'. El propósito central de este modelo educativo es que los niños lleguen a ser culturalmente competentes mediante la participación en prácticas culturales comunitarias. En esta propuesta, las lenguas son entendidas como medios para la socialización infantil. 
Los estudios arriba citados concuerdan en atribuir el escaso porcentaje de hablantes y -por ende- los bajos niveles de competencia en mapuzungun en la población infantil ${ }^{1}$ a una situación general de desplazamiento sociolingüístico, que se ve matizado por situaciones específicas a nivel territorial y actitudes lingüísticas variables en los entornos familiares de los niños. En relación a la dimensión territorial, las zonas rurales de La Araucanía muestran mayor heterogeneidad en los niveles de competencia infantil, dato que contrasta con la situación de niños de otras regiones o de zonas urbanas de la misma región (Gundermann et al., 2009 \& 2011; Wittig \& Olate, 2016). Lo anterior se relaciona con los hallazgos de Henríquez (2014) y Relmuan (2005) relativos a la localización de comunidades y territorios específicos en los cuales la población infantil presenta niveles de competencia en mapuzungun muy superiores a la media.

El entorno familiar-comunitario constituye otra dimensión relevante para el estudio de la competencia infantil en mapuzungun. Las investigaciones sociolingüísticas identifican dos aspectos críticos que inciden en los escasos niveles de competencia lingüística de la población infantil, a saber, el quiebre de la transmisión intergeneracional y las actitudes lingüísticas de los padres (Olate, Alonqueo \& Caniguan, 2013; Henríquez, 2015).

Tratándose de población infantil, la escuela es un contexto de desarrollo relevante por los procesos sociolingüísticos de interés para este estudio. Si bien es cierto que históricamente la escuela operó como un espacio de negación de la lengua y la identidad (Mansilla, Llancavil, Mieres \& Montanares, 2016), en las últimas décadas las políticas educacionales han cambiado su rumbo propiciando la inserción del mapuzungun en el currículum escolar, sobre todo en establecimientos de zonas rurales como los que forman parte de esta investigación.

No obstante, el conocimiento respecto del impacto de estas políticas y programas derivados en los procesos de adquisición y aprendizaje del mapuzungun es aún insuficiente. Para avanzar en este sentido, es necesario contar con estudios empíricos que aborden aspectos específicos de la competencia lingüística de los niños, como la caracterización de niveles de desempeño, la identificación de dimensiones lingüísticopragmáticas y la relación con el desarrollo cognitivo, entre otros. Por otro

En estos estudios solo se considera población infantil a partir de los 10 años. 
lado, el análisis de estas dimensiones no puede hacerse sin considerar las características específicas de las comunidades educativas a nivel lingüístico y sociocultural. Se requiere, en síntesis, la integración de estas dimensiones en un programa de investigación interdisciplinar que asuma una concepción situada de la competencia lingüístico-comunicativa y una mirada integral de los procesos de adquisición, aprendizaje y socialización infantil.

\section{Adquisición y aprendizaje de la competencia lingüístico-comunicativa}

El estudio de la competencia lingüístico-comunicativa tiene una larga tradición en lingüística y otras ciencias que se ocupan del lenguaje y la comunicación. En su desarrollo se pueden identificar transiciones desde enfoques centrados en los aspectos formales de la lengua y su codificación hacia formulaciones más contemporáneas en que lo lingüístico se concibe como una parte más de la competencia comunicativa, integrada además por dimensiones relativas a procesos cognitivos, pragmáticos y socioculturales, entre otros.

Para efectos del problema y el contexto en que se sitúa esta investigación, entendemos la competencia lingüístico-comunicativa en los términos propuestos por Saville-Troike (2005), es decir, como el conjunto de destrezas, conocimientos y actitudes que se interrelacionan en el acto de la comunicación. Lo anterior implica que los hablantes utilizan en mayor o menor grado sus habilidades lingüísticas, cognitivas y sociopragmáticas en función de propósitos comunicativos específicos y, a la vez, contextualizados. Tal como señala Hecht (2013), la adquisición de estas habilidades en hablantes infantiles es especialmente compleja en contextos sociolingüísticos indoamericanos, pues les exige movilizar conocimientos, valoraciones y pautas de interacción que se expresan en sistemas culturales y lingüísticos en contacto insertos relación asimétrica entre ellos.

Una revisión de la bibliografía relativa a la adquisición del mapuzungun muestra la escasez de estudios y su carácter fragmentario, ya que se abordan dimensiones muy específicas de la adquisición de la lengua y tramos etarios restringidos. Por ejemplo, el trabajo de Ibáñez (2015) se centra en el contexto interaccional entre lactantes ( 0 a 26 meses) y sus cuidadores en la etapa del desarrollo prelingüístico. Otro estudio se refiere al estado de la fonología del mapuzungun en adolescentes de 12 a 15 años (Henríquez, 2016). 
No obstante lo fragmentario del conocimiento empírico disponible, las políticas educativas proponen tres perfiles de competencia lingüística en la población infantil mapuche de las regiones del sur de Chile (Ministerio de Educación, 2011). Un perfil mayoritario correspondería a niños sin competencia previa en mapuzungun, y que entrarían en contacto con la lengua solo al ingresar al sistema escolar. Un segundo perfil equivaldría a niños que han desarrollado algún nivel de competencia, sobre todo a nivel de la comprensión. El último de los perfiles propuestos estaría representado por un grupo minoritario, circunscrito a territorios específicos, que ha desarrollado niveles suficientes de competencia en mapuzungun en la etapa pre-escolar.

Esta caracterización no cuenta con el debido respaldo empírico, sin embargo, es la base sobre la cual se ha diseñado el currículum para la enseñanza del mapuzungun en el contexto escolar. En este sentido, las escasas investigaciones que abordan la relación entre contexto escolar, aprendizaje y competencia lingüística en mapuzungun (ÁlvarezSantullano \& Forno, 2008; Quintrileo, Yáñez \& Valenzuela, 2013; Lagos, 2015; Luna et al., 2014) no han logrado configurar un conjunto sistemático y coherente que permita dimensionar sus impactos. A su vez, la implementación de los programas de enseñanza del mapuzungun han sido objeto de críticas que apuntan al enfoque adoptado para la enseñanza de la lengua, la falta de autonomía de los proyectos educativos, la adecuación de los materiales didácticos y, por último, la formación de los docentes y educadores tradicionales (Espinoza, 2016).

La complejidad inherente al desarrollo de estas habilidades en el contexto de estudio excede las posibilidades descriptivas de enfoques estructurales o normativos sobre las lenguas y la comunicación. De acuerdo con De León (2009), el marco teórico de la socialización lingüística (Ochs \& Schieffelin, 1995) permite abordar esta complejidad mediante investigaciones de corte etnográfico, otorgando mayor profundidad a la descripción de los procesos de adquisición de las lenguas indígenas en comunidades minorizadas. En este marco, la socialización lingüística se entiende como el resultado de las actividades socioculturales en las que los niños participan cotidianamente, considerando las lenguas a las que están expuestos y la estimulación explícita o implícita para aprenderlas. En otras palabras, se trata de dos procesos simultáneos que implican, por una parte, adquirir las lenguas como 'instrumentos' para la comunicación cotidiana y, por otra, socializarse o hacerse miembro de una comunidad 
a través un determinado repertorio de usos bi o multilingües, en sintonía con el planteamiento general del modelo educativo mapuche señalado más arriba.

En función de los antecedentes teóricos y empíricos presentados en los apartados previos, este artículo tiene como propósito describir la competencia lingüístico-comunicativa en mapuzungun en escolares mapuche de la región de La Araucanía. Este estudio aborda la evaluación de la competencia desde una perspectiva sociocultural que busca evitar un sesgo negativo en la medición a través de instrumentos y procedimientos culturalmente sensibles y pertinentes para la población de estudio (De Villiers \& De Villiers, 2010). De este modo, esta investigación se centra en la competencia oral del mapuzungun mediante una evaluación interactiva, con validez ecológica. La noción de competencia se operacionaliza en dimensiones lingüístico-comunicativa de complejidad creciente, que abarcan los procesos de comprensión y producción como aspectos interdependientes, pero con características específicas.

El objetivo general de este artículo es describir la competencia lingüística oral en mapuzungun de niños mapuche de cuatro escuelas rurales de la Región de La Araucanía. De manera más específica, pretende (i) evaluar niveles de competencia lingüística en comprensión y producción del mapuzungun en niños mapuche de cuatro escuelas rurales de la Región de La Araucanía; (ii) analizar los niveles de competencia en mapuzungun según los tramos etarios de los participantes.; y (iii) Analizar los niveles de competencia en mapuzungun según el establecimiento educativo de procedencia.

\section{Método}

El diseño de la investigación es de corte transeccional con alcance descriptivo.

\subsection{Participantes y contexto}

Mediante un muestreo por conveniencia se seleccionaron a 99 niños mapuche (52 niñas y 47 niños) de 6 a 10 años de edad de cuatro escuelas rurales de la Región de la Araucanía. Estas contaban con la implementación del Programa de Educación Intercultural Bilingüe y el Subsector de Lengua Indígena. 
La Escuela 1 está ubicada en un sector rural de la comuna de Angol distante a 25 kilómetros de esta ciudad. Es un establecimiento municipal que cuenta con la enseñanza básica completa de $1^{\circ}$ a $8^{\circ}$ año. En esta zona se destaca la alta presencia de plantaciones forestales y conflictos territoriales derivados de aquello.

La Escuela 2 se encuentra localizada en un sector rural de Boroa distante a 9 kilómetros de la ciudad de Nueva Imperial. Es un establecimiento particular subvencionada de orientación confesional católica que imparte enseñanza desde $1^{\circ}$ a $8^{\circ}$ básico. Cuenta con una amplia infraestructura que incluye una residencia con régimen de internado para los estudiantes durante el periodo escolar.

La Escuela 3 está ubicada en la localidad de General López, distante a 13 kilómetros de la ciudad de Vilcún. El establecimiento es de dependencia particular subvencionada e imparte enseñanza básica desde $1^{\circ}$ a $8^{\circ}$ año.

La Escuela 4 se encuentra en el sector de Truf Truf distante a 7 kilómetros de la zona urbana de Padre Las Casas. Es un establecimiento particular subvencionado de orientación cristiana que imparte enseñanza básica y media de tipo técnico-profesional.

La selección de los participantes se hizo en base a dos criterios de inclusión: a) autoidentificación mapuche y b) residencia en una comunidad mapuche. Como criterios de exclusión, se consideró la participación en programas de integración escolar y un rezago escolar superior a 2 años.

\subsection{Instrumento}

Se aplicó un instrumento evaluativo de elaboración ad hoc denominado Cuestionario de Competencia Lingüística en Mapuzungun (CCLM)². Una descripción detallada de este instrumento se presenta en Alonqueo, Wittig y Huenchunao (2017). Este instrumento evalúa comprensión y producción en mapuzungun y se compone de 7 secciones: Léxico, Saludos, Directivos, Términos Espaciales, Numéricos y Temporales, y Narración. Cada una de estas incluye un número variable de preguntas distribuidas en 3 niveles de complejidad: baja, media y alta. Además, contiene una

Este instrumento fue elaborado por el equipo de investigación del proyecto Fondecyt $\mathrm{N}^{\circ} 1130504$ y del proyecto DIUFRO DI12-0005. 
sección de datos personales y 4 preguntas relativas a características generales de la familia del niño: participación en el ngillatun, presencia de hablantes de mapuzungun, religión y uso de sistemas médicos.

\subsection{Análisis de la información}

El instrumento cuenta con un manual de puntuación en el que se definen ejemplos y rúbricas de respuesta a partir de las cuales se asigna puntaje a las respuestas que pueden recogerse en todos los ítems. Cada participante obtiene una puntuación total ponderada para comprensión y para producción, lo que permite asignarlos a una de las 6 categorías de competencia lingüística. Con ello se establece una relación entre el rendimiento alcanzado por el participante y los niveles de competencia lingüístico-comunicativa en mapuzungun, como se indica en la Tabla 1.

Tabla 1. Niveles de competencia lingüística.

\begin{tabular}{|l|c|l|}
\hline $\begin{array}{c}\text { Nivel de } \\
\text { Competencia }\end{array}$ & Puntaje & \multicolumn{1}{c|}{ Dimensión lingüístico-comunicativa } \\
\hline Nula & 0 & - \\
\hline Baja inferior & $1-15$ & Léxico y saludos \\
\hline Baja superior & $16-30$ & Baja inferior + directivos \\
\hline Media inferior & $31-45$ & $\begin{array}{l}\text { Baja superior + Términos espaciales o temporales o } \\
\text { numéricos }\end{array}$ \\
\hline Media Superior & $46-65$ & $\begin{array}{l}\text { Baja superior + Términos espaciales y temporales y } \\
\text { numéricos }\end{array}$ \\
\hline Alta & $66-100$ & Media superior + Narración \\
\hline
\end{tabular}

\subsection{Procedimiento}

El instrumento fue aplicado individualmente por una profesora básica, hablante nativa de la lengua. Las consignas generales se entregaron en español, mientras que los ítems de prueba se formularon íntegramente en mapuzungun. Cada ítem se presentó en dos instancias, ambas en mapuzungun. La administración del cuestionario tuvo una duración aproximada de 30 minutos. Las respuestas de los participantes fueron registradas en formato de audio y por escrito. Una vez finalizada la aplicación del instrumento se retribuyó a cada participante con una colación y materiales escolares. 
Se tomaron los debidos resguardos éticos mediante el consentimiento informado de los padres de los escolares y el asentimiento de estos últimos por medio de la firma de la documentación pertinente.

\section{Resultados}

\subsection{Caracterización familiar de los participantes}

En primer lugar, se presentará un resumen de las características de las familias descritas desde la perspectiva de los propios niños.

Sobre la participación familiar en el ngillatun un poco más de la mitad de los niños (53\%) refiere que sus familias participan de esta práctica sociorreligiosa, mientras que el $47 \%$ restante señala que su familia no asiste. Por otra parte, un $\mathbf{8 8 \%}$ de los participantes declara que en sus hogares existen hablantes competentes del mapuzungun en la generación de los abuelos y/o los padres. Solo un $12 \%$ de los participantes refiere la ausencia de hablantes de la lengua en su grupo familiar. También se indagó la adscripción religiosa de las familias obteniendo que un $39 \%$ de los participantes declara que pertenece a la religión evangélica, mientras que un $30 \%$ señala que solo tienen creencias mapuche. La religión católica está presente en un 16\% de las familias, y el restante $14 \%$ de los niños reporta desconocer la filiación religiosa de su familia. Por último, para el tratamiento de las enfermedades el sistema médico más recurrido es la medicina occidental (40\%) seguida de la combinación de medicina mapuche y occidental (30\%). Un 16\% de los niños declara que solo recurren a la machi, y un $9 \%$ a la herbolaria casera. Un número menor de respuestas señala el uso de oraciones religiosas (3\%), y solo $1 \%$ declara desconocer dicha información.

Se deriva de esta información una caracterización sociocultural heterogénea. En relación al escenario sociolingüístico regional y nacional, existe un alto porcentaje de hablantes en el entorno familiar inmediato de los niños. Ello implica que están expuestos -en mayor o menor medida- a un input lingüístico en mapuzungun independiente de si se verifica o no el desarrollo de una competencia activa en los niños. En relación a las dimensiones socioculturales, hay una participación importante en prácticas religiosas y culturales que son centrales para el modo de vida mapuche (Catriquir \& Durán, 2007; Quidel \& Pichinao, 2007). Lo heterogéneo se observa en la presencia de prácticas religiosas 
y culturales no mapuche, como el uso de medicina occidental y las religiones judeocristianas. En esta investigación, asumimos que estas características socioculturales son resultado de las relaciones de contacto cultural y su trasfondo histórico local. (Nahuelpán, 2012).

\subsection{Niveles de competencia en producción y comprensión}

Se presentan los resultados obtenidos por la totalidad de la muestra en las dimensiones de comprensión y producción. Los datos que se muestran en la Tabla 2 indican un bajo desempeño general en la lengua mapuche, ya que la gran mayoría de los participantes evidencia un nivel de competencia Baja Inferior ( $86 \%$ en comprensión y 85\% en producción). Es destacable que los pocos casos que muestran un mayor nivel de competencia obtienen mejores resultados en comprensión alcanzando niveles de competencia media superior y alta. Sin embargo, al analizar la producción de estos mismos niños se obtiene que ninguno de ellos alcanzó un nivel de competencia alta.

Tabla 2. Niveles de Competencia en Comprensión y Producción.

\begin{tabular}{|l|c|c|}
\hline \multicolumn{1}{|c|}{ Nivel de Competencia } & $\begin{array}{c}\text { Comprensión } \\
\mathbf{\%} \mathbf{f}\end{array}$ & $\begin{array}{c}\text { Producción } \\
\mathbf{\%} \mathbf{f}\end{array}$ \\
\hline Nula & $1(1)$ & $5(5)$ \\
\hline Baja inferior & $86(85)$ & $85(84)$ \\
\hline Baja superior & $4(4)$ & $5(5)$ \\
\hline Media inferior & $1(1)$ & $5(5)$ \\
\hline Media superior & $6(6)$ & 0 \\
\hline Alta & $2(2)$ & 0 \\
\hline
\end{tabular}

Para efectos de este estudio, los resultados generales se analizaron en función de dos variables importantes, a saber, la edad de los participantes y el establecimiento educativo.

Sin embargo, un análisis más detallado de los datos permite identificar algunos matices en relación con la edad de los participantes. En las tablas 3 y 4 se muestra la distribución de los niveles de competencia en mapuzungun según la edad de los participantes. En la dimensión 'compresión', los resultados obtenidos por la totalidad de los niños menores de 8 años dan cuenta de un nivel de competencia baja inferior (Tabla 3). En los tramos de edad siguientes, si bien se mantienen la tendencia general, al menos los participantes muestran mejores 
desempeños, alcanzando incluso niveles de competencia media superior (4 de los casos) y alta (solo 1 caso).

Tabla 3. Nivel de Competencia en Comprensión según edad.

\begin{tabular}{|c|c|c|c|c|c|c|c|}
\hline $\begin{array}{c}\text { Competencia } \\
\text { Edad }\end{array}$ & $\mathbf{N}$ & $\begin{array}{l}\text { Nula } \\
\% \text { (f) }\end{array}$ & $\begin{array}{c}\text { Baja } \\
\text { Inferior } \\
\% \text { (f) }\end{array}$ & $\begin{array}{c}\text { Baja } \\
\text { Superior } \\
\text { \% (f) }\end{array}$ & $\begin{array}{c}\text { Media } \\
\text { Inferior } \\
\% \text { (f) }\end{array}$ & $\begin{array}{c}\text { Media } \\
\text { Superior } \\
\text { \% (f) }\end{array}$ & $\begin{array}{l}\text { Alta } \\
\%(f)\end{array}$ \\
\hline $6 ; 0$ a 6;11 & 14 & $\mathrm{o}(\mathrm{o})$ & $100(14)$ & $\mathrm{o}(\mathrm{o})$ & $o(0)$ & $o(0)$ & $\mathrm{o}(\mathrm{o})$ \\
\hline 7;0 a 7;11 & 20 & $\mathrm{o}(\mathrm{o})$ & $100(20)$ & $\mathrm{o}(\mathrm{o})$ & $\mathrm{o}(\mathrm{o})$ & $\mathrm{o}(\mathrm{o})$ & $\mathrm{o}(\mathrm{o})$ \\
\hline 8;0 a 8;11 & 24 & $4,2(1)$ & $83,3(20)$ & $\mathrm{o}(\mathrm{o})$ & $4,2(1)$ & $8,3(2)$ & $\mathrm{o}(\mathrm{o})$ \\
\hline 9;0 a 9;11 & 28 & $\mathrm{o}(\mathrm{o})$ & $71,4(20)$ & $10,7(3)$ & $\mathrm{o}(\mathrm{o})$ & $14,3(4)$ & $3,6(1)$ \\
\hline 10;0 a 10;11 & 13 & $\mathrm{o}(\mathrm{o})$ & $84,6(11)$ & $7,7(1)$ & $\mathrm{o}(\mathrm{o})$ & $\mathrm{o}(\mathrm{o})$ & $7,7(1)$ \\
\hline Total & 99 & & & & & & \\
\hline
\end{tabular}

Tabla 4. Nivel de Competencia en Producción según edad.

\begin{tabular}{|c|c|c|c|c|c|c|c|}
\hline $\begin{array}{c}\text { Competencia } \\
\text { Edad }\end{array}$ & $\mathbf{N}$ & $\begin{array}{l}\text { Nula } \\
\% \text { (f) }\end{array}$ & $\begin{array}{c}\text { Baja } \\
\text { Inferior } \\
\% \text { (f) }\end{array}$ & $\begin{array}{c}\text { Baja } \\
\text { Superior } \\
\% \text { (f) }\end{array}$ & $\begin{array}{c}\text { Media } \\
\text { Inferior } \\
\% \text { (f) }\end{array}$ & $\begin{array}{c}\text { Media } \\
\text { Superior } \\
\% \text { (f) }\end{array}$ & $\begin{array}{l}\text { Alta } \\
\%(f)\end{array}$ \\
\hline 6;0 a 6;11 & 14 & $7,1(1)$ & $92,9(13)$ & $o(0)$ & $\mathrm{o}(\mathrm{o})$ & $o(0)$ & $\mathrm{o}(\mathrm{o})$ \\
\hline 7;0 a 7;11 & 20 & $5,0(1)$ & $95,0(19)$ & $o(0)$ & $\mathrm{o}(\mathrm{o})$ & $\mathrm{o}(\mathrm{o})$ & $\mathrm{o}(\mathrm{o})$ \\
\hline $8 ; 0$ a 8;11 & 24 & $4,2(1)$ & $83,3(20)$ & $\mathrm{o}(0)$ & $12,5(3)$ & $\mathrm{o}(\mathrm{o})$ & $\mathrm{o}(\mathrm{o})$ \\
\hline 9;0 a 9;11 & 28 & $3,6(1)$ & $76,6(22)$ & $14,2(4)$ & $3,6(1)$ & $o(0)$ & $\mathrm{o}(\mathrm{o})$ \\
\hline 10;0 a 10;11 & 13 & $7,7(1)$ & $76,9(10)$ & $7,7(1)$ & $7,7(1)$ & $\mathrm{o}(\mathrm{o})$ & $\mathrm{o}(\mathrm{o})$ \\
\hline Total & 99 & & & & & & \\
\hline
\end{tabular}

Como se puede apreciar en la Tabla 4, la tendencia antes descrita también se corrobora en los datos de producción, es decir, son los niños mayores de 8 años quienes exhiben un mejor dominio de la lengua mapuche. Pero en esta dimensión se observa una mayor cantidad de niños con competencia nula (5 casos), y ningún caso con niveles de competencia media superior y alto.

Por otra parte, al analizar los resultados de los niños según su establecimiento de procedencia, se observan resultados interesantes. Tal como se puede apreciar en las Tablas 5 y 6 , una primera mirada a los resultados muestra que las cuatro escuelas comparten la tendencia general que señala la existencia de una baja competencia en la lengua mapuche. Específicamente, dos de los establecimientos (Escuelas 1 y 2) muestran resultados prácticamente idénticos, concentrando la totalidad de los participantes en el nivel de competencia baja inferior 
para comprensión, y competencia nula y baja inferior para producción. En tanto, las escuelas 3 y 4 presentan un mayor grado de variabilidad en los desempeños de los niños en las dos dimensiones evaluadas. En estos establecimientos se pueden identificar casos específicos de niños con niveles de competencia que van desde baja superior hasta alta, especialmente en comprensión.

Tabla 5. Nivel de competencia en comprensión según escuela.

\begin{tabular}{|c|c|c|c|c|c|c|c|}
\hline $\begin{array}{l}\text { Competencia } \\
\text { Escuela }\end{array}$ & $\mathbf{N}$ & $\begin{array}{l}\text { Nula } \\
\% \text { (f) }\end{array}$ & $\begin{array}{c}\text { Baja } \\
\text { Inferior } \\
\% \text { (f) }\end{array}$ & $\begin{array}{c}\text { Baja } \\
\text { Superior } \\
\text { \% (f) }\end{array}$ & $\begin{array}{c}\text { Media } \\
\text { Inferior } \\
\% \text { (f) }\end{array}$ & $\begin{array}{c}\text { Media } \\
\text { Superior } \\
\%(f)\end{array}$ & $\begin{array}{l}\text { Alta } \\
\%(f)\end{array}$ \\
\hline 1 & 19 & $\mathrm{o}(\mathrm{o})$ & $100(19)$ & $\mathrm{o}(\mathrm{o})$ & $o(0)$ & $\mathrm{o}(\mathrm{o})$ & $\mathrm{o}(\mathrm{o})$ \\
\hline 2 & 14 & $\mathrm{o}(\mathrm{o})$ & $100(14)$ & $\mathrm{o}(\mathrm{o})$ & $\mathrm{o}(\mathrm{o})$ & $\mathrm{o}(\mathrm{o})$ & $\mathrm{o}(\mathrm{o})$ \\
\hline 3 & 31 & $3,2(1)$ & $71(22)$ & $6,5(2)$ & $9,7(3)$ & $6,5(2)$ & $3,2(1)$ \\
\hline 4 & 34 & $\mathrm{o}(\mathrm{o})$ & $85,3(29)$ & $\mathrm{o}(\mathrm{o})$ & $5,9(2)$ & $5,9(2)$ & $2,9(1)$ \\
\hline Total & 99 & & & & & & \\
\hline
\end{tabular}

Tabla 6. Nivel de competencia en producción según escuela.

\begin{tabular}{|c|c|c|c|c|c|c|c|}
\hline $\begin{array}{l}\text { Competencia } \\
\text { Escuela }\end{array}$ & $\mathbf{N}$ & $\begin{array}{l}\text { Nula } \\
\% \text { (f) }\end{array}$ & $\begin{array}{c}\text { Baja } \\
\text { Inferior } \\
\%(f)\end{array}$ & $\begin{array}{c}\text { Baja } \\
\text { Superior } \\
\%(f)\end{array}$ & $\begin{array}{c}\text { Media } \\
\text { Inferior } \\
\% \text { (f) }\end{array}$ & $\begin{array}{c}\text { Media } \\
\text { Superior } \\
\% \text { (f) }\end{array}$ & $\begin{array}{l}\text { Alta } \\
\%(f)\end{array}$ \\
\hline 1 & 19 & $5,3(1)$ & $94,7(18)$ & $\mathrm{o}(\mathrm{o})$ & $\mathrm{o}(\mathrm{o})$ & $\mathrm{o}(\mathrm{o})$ & $\mathrm{o}(\mathrm{o})$ \\
\hline 2 & 14 & $14,3(2)$ & $85,7(12)$ & $\mathrm{o}(\mathrm{o})$ & $\mathrm{o}(\mathrm{o})$ & $\mathrm{o}(\mathrm{o})$ & $\mathrm{o}(\mathrm{o})$ \\
\hline 3 & 31 & $6,5(2)$ & $71,0(22)$ & $16,0(5)$ & $6,5(2)$ & (o) & (o) \\
\hline 4 & 34 & $o(0)$ & $91,2(31)$ & $\mathrm{o}(\mathrm{o})$ & $8.8(3)$ & (o) & (o) \\
\hline Total & 99 & & & & & & \\
\hline
\end{tabular}

\section{Discusión}

De acuerdo con los resultados generales del estudio, la competencia lingüístico-comunicativa en mapuzungun de los participantes corresponde a habilidades de nivel bajo, básicamente expresiones de saludo y unidades léxicas, con un mayor predominio de habilidades receptivas por sobre las productivas. Este panorama general presenta algunas variaciones asociadas a los tramos de edad y escuelas de procedencia de los participantes. Concretamente, un número acotado de participantes entre 8 y 10 años y procedentes de dos de las cuatro escuelas consideradas en el estudio mostró desempeños de nivel medio y alto -este último nivel solo en comprensión-. En los párrafos siguientes, se propone un 
análisis de estos resultados en torno a dos procesos complementarios: uno de orden evolutivo (adquisición y desarrollo de la competencia en mapunzungun) y el otro de orden sociocultural (prácticas familiares y socialización lingüística).

Respecto del proceso de orden evolutivo, los resultados muestran que, hasta los 8 años, el nivel de competencia en mapuzungun se mantiene constante en un nivel bajo, con un manejo relativo de unidades léxicas y expresiones de saludo. Como complemento de lo anterior, los casos de competencia media y alta corresponden solo a niños entre 8 y 10 años. En el análisis cualitativo de una parte de esta muestra, el desempeño de los niños de 8 a 10 años con competencia media o alta se caracterizó por la comprensión de componentes específicos de la lengua (directivos, términos numéricos, espacio-temporales y narración), además de habilidades productivas en léxico y saludos (ver Alonqueo, Wittig \& Huenchunao, 2017).

Dadas las características descriptivas de esta investigación, solo es posible hacer planteamientos preliminares respecto de la relación entre tramos de edad y desarrollo de la competencia lingüísticocomunicativa. Para avanzar hacia formulaciones de mayor poder explicativo, se requieren estudios longitudinales con muestras de mayor representatividad que describan el proceso de adquisición de la lengua en paralelo al desarrollo evolutivo de los niños. Del mismo modo, se requieren investigaciones etnográficas que permitan comprender el proceso situándolo en las pautas de interacción comunicativa de los niños en sus contextos familiar, escolar y comunitario.

Respecto del proceso sociocultural, los datos obtenidos sobre la presencia de hablantes entre los familiares directos y su participación en prácticas culturales tradicionales que implican el uso de la lengua sugieren en principio una exposición cotidiana al mapuzungun en el entorno familiar y comunitario de los niños. No obstante, y tal como se ha planteado en otros estudios (Wittig, 2011; Olate et al., 2013; Henríquez, 2015; Huenchunao, 2015) la presencia de adultos hablantes no implica necesariamente que estos se dirijan a los niños en mapuzungun en estas interacciones cotidianas. De momento no es posible señalar si esta forma de interacción responde a una decisión deliberada respecto de la transmisión o no transmisión de la lengua. Para profundizar en este asunto habría que orientar el análisis al estudio de las actitudes lingüísticas de padres y abuelos (o cuidadores) y cómo estas permean 
las prácticas cotidianas que propician, dificultan o retrasan la transmisión intergeneracional de la lengua. Un asunto relevante consiste en indagar en las posibles relaciones entre las actitudes lingüísticas de grupos familiares específicos, por un lado, y los discursos y prácticas relativos a la lengua en el nivel de la comunidad u otras instancias sociopolíticas relevantes en una perspectiva propia mapuche, por otro.

A pesar de las limitaciones inherentes al alcance descriptivo de la investigación, es posible sostener a partir de los planteamientos anteriores un proceso de adquisición del mapuzungun tardío respecto del castellano o secuencial entre una y otra lengua en el entendido de que ambas forman parte de un repertorio sociolingüístico en el que se privilegia la funcionalidad comunicativa del castellano en los contextos familiar y escolar, mientras que el uso del mapuzungun se favorece en contextos intraculturales específicos y de modo gradual una vez que los niños van adquiriendo otras competencias culturales primero.

Considerando que el estudio se centra en población infantil, el análisis de los resultados incluye el papel de la escuela en el aprendizaje formal del mapuzungun, aun cuando esta dimensión no sea central al objetivo de la investigación. En este sentido, el hallazgo central es la concentración de los niveles de competencia media y alta en niños de dos de las cuatro escuelas participantes. Este dato debe situarse en relación con las características particulares de cada comunidad educativa y los territorios en los cuales están situadas. La dimensión territorial adquiere mayor relevancia si se toma en cuenta que en todas las escuelas se implementa el programa nacional de enseñanza formal del mapuzungun. Las posibilidades interpretativas quedan abiertas en la medida de que este estudio no se propone indagar sobre prácticas pedagógicas ni didáctica específica del mapuzungun en contexto escolar. Una posibilidad es que el programa, en términos generales no esté logrando los aprendizajes esperados en la dimensión lingüísticocomunicativa, relación que a la fecha solo ha sido abordada de manera tangencial (Espinoza 2016; Luna et al. 2014). Una segunda posibilidad apunta a las diferencias internas entre los distintos establecimientos, sobre todo teniendo en cuenta que las prácticas pedagógicas no son neutras, sino que están intencionadas desde lineamientos institucionales, en la línea de lo señalado por Álvarez-Santullano y Forno (2008) e Ibáñez (2015). Una tercera posibilidad apunta a las dinámicas de interacción entre la escuela, la familia y la comunidad, 
tal como se sugiere en Ibáñez (2015), Espinoza (2016) y Mansilla et al. (2016).

Futuros trabajos debieran explorar la influencia de la escuela en la revitalización de la lengua y la interrelación entre familia, comunidad y escuela en este proceso de adquisición y socialización lingüísticocultural. Un análisis comprehensivo de esta intersección permitirá orientar de mejor manera las políticas y prácticas de revitalización lingüística enfocadas en la población infantil mapuche.

\section{Referencias bibliográficas}

Alonqueo, P., Wittig, F. y Huenchunao, N. (2017). Lleupeko tuwün:Un estudio exploratorio sobre niveles de competencia en mapuzungun en niños mapuche de la Araucanía. Revista Alpha, 44, 119-135.

Álvarez-Santullano, P., \& Forno, A. (2008). La inserción de la lengua mapuche en el currículum de escuelas con educación intercultural: un problema más que metodológico. Alpha, 26, 9-28.

Catriquir, D., \& Durán, T. (2007). Kimeltuwün zugu: Modelo educativo mapunche. En D. C. T. Durán, Patrimonio cultural mapunche. Volumen III (pp. 443-454). Temuco: Universidad Católica de Temuco.

De León, L. (2009). La llegada del alma. México: Casa Chata.

De Villiers, P., \& De Villiers, J. (2010). Assessment of language acquisition. WIREs Cognitve Sciences, 1, 230-244.

Espinoza, M.(2016). Contextos, metodologías y duplas pedagógicas en el Programa de Educación Intercultural Bilingüe en Chile: una evaluación crítica del estado del debate. Pensamiento Educativo. Revista de Investigación Educacional Latinoamericana, 53(1), 1-16.

Gundermann, H., Canihuán, J., Clavería, A., \& Faúndez, C. (2009). Permanencia y desplazamiento, hipótesis acerca de la vitalidad del mapuzugun. RLA Revista de Lingüística Teórica y Aplicada, 47(1), 37-60.

(2011). El mapuzugun, una lengua en retroceso. Atenea, 503, 101-131. 
Hecht, A. (2013). Niñez, escolarización y lengua indígena. Una mirada antropológica sobre la diversidad lingüística en la escuela. Revista de Educacao Publica, 22, 405-419.

Henríquez, M. (2014). Estado del mapudungun en comunidades pewenches y lafkenches de la región del Bío-Bío: el caso de los escolares. RLA Revista de Lingüística Teórica y Aplicada, 52(2), 13-40.

- (2015). Ámbitos de uso del mapudungun en comunidades pewenches y lafkenches de la Región del Bío-Bío. Literatura y Lingüística, 31, 185-204.

. (2016). Estado de la fonología segmental del mapudungun de escolares lafkenches de la comuna de Tirúa: rasgos prominentes. Literatura y Lingüística, 34, 295-318.

Henríquez, M., \& Salamanca, G. (2015). Vitalidad de la fonología segmental del chedungun hablado por escolares del Alto BioBio. Alpha, 41, 207-231.

Huenchunao, N. (2015). Transmisión del mapudungun en la interacción familiar cotidiana y competencia lingüistica infantil: Un estudio en escolares mapuche de la novena región. Tesis de Magíster, Universidad de La Frontera, Chile.

Ibáñez Salgado, Nolfa. (2015). La tríada cultural-contextual: Una oportunidad para asegurar la pertinencia cultural en la implementación de la educación intercultural. Estudios pedagógicos (Valdivia), 41(1), 323-335.

Lagos, C. (2012). Mapudungún en Santiago de Chile: representaciones sociales en los mapuches urbanos. RLA. Revista de Lingüística Teórica y Aplicada, 50(1), 161-190.

. (2015). El programa de educación bilingüe y sus resultados: ¿perpetuando la discriminación? . Pensamiento Educativo. Revista de Investigación Educacional Latinoamericana, 52(1), 84-94.

Luna, L., Benavides, P., Gutiérrez, P., Alchao, M., \& Dittborn, A. (2014). Aprender lengua y cultura mapuche en la escuela: estudio de caso de la implementación del nuevo Sector de Aprendizaje Lengua Indígena desde un análisis de «recursos educativos". Estudios Pedagógicos, 40(20), 221-240. 
Mansilla, J., Llancamil, D., Mieres, M., \& Montanares, E. (2016). Instalación de la escuela monocultural en la Araucanía, 18831910: dispositivos de poder y Sociedad Mapuche. Educaçao e Pesquisa, 42, 213-228.

Ministerio de Educación (2011). PEIB-ORÍGENES estudio sobre la implementación de la Educación Intercultural Bilingüe. Santiago: MInisterio de Educación.

Nahuelpán, H. (2012). Formación colonial del estado y desposesión en Ngulumapu. En Comunidad de Historia Mapuche. Ta iñ fijke xipa rakizuameluwun: Historia, colonialismo y resistencia desde el país Mapuche (pp. 123-156). Temuco: Ediciones Comunidad de Historia Mapuche.

Ochs, E., \& Shieffelin, B. (1995). The socialization on grammatical development. En P. Fletcher and MacWhinney, The Handbook of Child Languag (pp. 73-94). Oxford: Blackwell.

Olate, A., Alonqueo, P., \& Canihuán, J. (2013). Interactividad lingüística castellano/mapudungun de una comunidad rural bilingüe. Alpha, 13, 265-284.

Quidel, J., \& Pichinao, J. (2007). Txemümkagen püchükeche mapun kimeltunmew. Formación de los niños y niñas en la educación mapunche. En T. C. Durán, Patrimonio cultural mapunche. Volumen III (pp. 1-2). Universidad Católica de Temuco: Temuco.

Quilaqueo, D., \& Quintriqueo, S. (2010). Saberes educativos mapuches: un análisis desde la perspectiva de los kimches. Polis Revista Latinoamericana, 9(26), 337-360.

Quintrileo, C., Yáñez, C., \& Valenzuela, C. (2013). Una aproximación crítica a la propuesta en consulta del Programa de Educación Intercultural Bilingüe (PEIB) en Chile. Logos: Revista de Lingüística, Filosofía y Literatura, 23(1), 45-61.

Relmuán, M. (2005). El contexto de uso en seis tipos de discurso mapuche y su posible inserción en el aula y la formación docente. Cochabamba: Universidad Mayor de San Simón.

Saville-Troike, M. (2005). Etnografía de la comunicación. Buenos Aires: Prometeo Libros.

Wittig, F. (2011). Adquisición y transmisión del mapudungún en hablantes urbanos. Literatura y lingüistica, 23, 193-211. 
Wittig, F., \& Olate, A. (2016). El mapuzugun en La Araucanía. Apuntes en torno al desfase entre la politización de la lengua y la heterogeneidad sociolingüística local. Universos, 13, 119-134.

Zúñiga, F. (2007). Mapudunguwelaymi am? Acaso ya no hablas mapudungun? Acerca del estado actual de la lengua mapuche. Centro de Estudios Públicos, 105, 9-25.

Zúñiga, F., \& Olate, A. (2017). El estado de la lengua mapuche, diez años después. En I. G. Aninat, El pueblo mapuche en el siglo XXI: propuestas para un nuevo entendimiento entre culturas en Chile (pp. 343-374). Santiago: Centro de Estudios Públicos. 